\title{
An Algebraic Theory of Portfolio Allocation
}

August 2001

by

David A. Hennessy

Professor

Department of Economics

Iowa State University

Ames, IA 50011-1070
Harvey E. Lapan

University Professor

Department of Economics

Iowa State University

Ames, IA 50011-1070

Mailing address for correspondence

David A. Hennessy

Department of Economics

478 B Heady Hall

Iowa State University

Ames, IA 50011-1070

$\mathrm{Ph}:$ (515) 294-6171

Fax: (515) 294-0221

e-mail: hennessy@iastate.edu 


\section{An Algebraic Theory of Portfolio Allocation}

Summary. Diversification, a central issue in the study of capital allocation, has much to do with symmetries and asymmetries in the distribution of asset returns. A diversified portfolio imposes symmetry on the allocation vector in order to balance out much of the asymmetries in the returns vector. Using group and majorization theory, we explore what can be established about the allocation vector when the asymmetries in the returns vector are carefully controlled. The key insight is that preferences over allocations can be partially ordered via majorized convex hulls that have been generated by group elements. It is shown that transitive permutation groups, rather than the more structured permutation symmetric group, suffice to ensure complete portfolio diversification. Point-wise stabilizer subgroups admit separability in the allocation of funds across sectors. When, together with imperfect symmetry in the sources of randomness, asset returns differ by heterogeneity in location or scale parameters then we bound the admissible allocation vector by a set of linear constraints. For a distribution that is symmetric under reflection groups, the linear constraints may be further strengthened whenever there exists an hyperplane that separates convex sets.

Keywords and Phrases: allocation vector, convex hull, group majorization, permutation group, pointwise stabilizer subgroup, reflection group, separability, transitive group

JEL classification: G0, D0, C6 


\section{Introduction}

It has long been noted that symmetries can be exploited to learn more about optimality in portfolio allocation decisions. Symmetries can be employed in many ways when specifying the problem. For instance, independence among returns imposes a very restrictive form of symmetry on stochastic interactions. The classical CAPM model, due to Sharpe (1964) among others, imposes the assumption of multivariate normality so that all uni-dimensional marginals are symmetric up to location and scale parameters and all stochastic interactions are also linear in form. Samuelson (1967a, 1967b), Brumelle (1974), Hadar, Russell and Seo (1977), McEntire (1984), Landsberger and Meilijson (1990), Kijima and Ohnishi (1996), Kijima (1997), and Lapan and Hennessy (2001) have all identified symmetries of various forms and strengths that are necessary, sufficient, or both when seeking to assert something about the optimal allocation vector for a risk averse expected utility maximizing investor.

Our concern with this literature is that, while insightful, rigorous, and ultimately of undoubted assistance to financial practitioners, the literature has not broached the issue of symmetry head-on. Mathematics has developed a variety of tools, particularly those arising from group and majorization theories, that are well suited to modeling symmetry and departures from symmetry. These tools have found widespread uses in other disciplines, such as physics and chemistry.

In economics explicit use of group theory has been confined to a few topics, such as Saari's (e.g., 2000a, 2000b) work on voting theory, and studies by Sato (1976), Russell and Farris (1998) and others on duality in production and consumption. Majorization tools, which have a somewhat indirect group theoretic underpinning, have found a limited but growing variety of uses in economics. The baseline form of majorization has been applied in work by Atkinson (1970) on social equity, work by Rothschild and Stiglitz (1970) on the welfare and comparative statics effects of risk, and work by Salant and Shaffer (1999) on heterogeneity in oligopoly. Chambers and Quiggin (2000) have engaged a generalized weighted extension of majorization in a variety of contexts concerning the theory of the firm when facing endogenous production uncertainty.

Given the natural symmetries embodied in the structure of the portfolio allocation problem, it would seem that the problem should be as amenable, if not more so, to the mathematical tools that have been 
designed to model symmetries. This paper will demonstrate that, even using basic concepts in group and majorization theories, much can be established about optimal fund allocation vectors. After first characterizing our specification of the portfolio problem, we will present the tools that will be used. The first analysis section, Section 4, applies a variant of majorization to identify the property of transitivity in a permutation group as sufficient to motivate complete diversification in a risk averter's portfolio. Given the wide variety of investment opportunities that are now typically available to investors in developed economies, it would be convenient to understand when a partial analysis, i.e., involving a subset of those opportunities, does not mis-represent the problem. Section 5 shows the utility of point-wise stabilizer groups in this regard.

Sections 6 and 7 introduce location and scale asymmetries, in addition to incompleteness in the symmetries among the sources of risk. By way of majorization under group operations and use of revealed preference arguments, we develop sets of linear inequalities that an optimal allocation vector must satisfy. We show how the resulting linear programming problem might be of use to practicing financial professionals. Section 8 demonstrates the particular convenience of reflection groups when applying revealed preference arguments. It is shown that, regardless of what else a group does, if a group element folds the distribution of risks back on itself such that the only difference between one set of assets and the reflected set is given by location parameters along a ray then the allocations are ordered as intuition would suggest. A corresponding inference is also valid when the source of differentiation arise from scale, rather than location, parameters. The paper concludes with some conjectures on strengthening our findings.

\section{Portfolio allocation problem}

A von-Neumann \& Morgenstern expected utility maximizing investor has a fixed amount of wealth, say $\$ 1$, available to invest at time 0 . She allocates it between $n$ available investment assets that provide time 1 gross returns $\$ x_{i}$ per $\$$ invested in opportunity, $i 0\{1,2, \ldots, n\}$ ' $\mathrm{O}_{n}$. The optimization problem $(P)$ may be represented as 


$$
\operatorname{Max}_{\mathrm{R} O \mathrm{~S}} E[U(\mathrm{p})], \quad \mathrm{p}{ }^{\prime} \mathbb{P} \Phi, \quad \text { s.t. } \mathbb{P} \mathbb{C}(1,
$$

with $\mathbb{R}^{\prime}\left(a_{1}, a_{2}, \ldots, a_{n}\right)$ and $S^{\prime}\left\{\mathbb{R}: \mathbb{P} \cdot \mathbf{P}^{\prime} 1, a_{i} 0[0,1]\right.$ ú $\left.i 0 \mathrm{O}_{n}\right\} .^{1}$ The '@onotation refers to the usual inner product operation. At present we assume that the vector of gross returns, $\mathbf{P}$, is nonnegative, i.e., $\mathbf{P O}{ }_{\%}^{*}$. The expectation operator, $E$ [Ф is with respect to the probability measure of $\mathbf{P}$ over the distribution $F(\mathbb{P})$. The conditions on $S$ suffice to ensure the existence of a solution to $(P)$. Our exclusive concern is with developing inferences on the ordinal and cardinal properties of solution vectors, $\mathbb{P}^{(}$, to problem $(P)$. We identify by $U_{1}^{(}$the set of agents solving $(P)$ who have a monotone non-decreasing utility function $U(\mathrm{p})$. And the subset of $U_{1}^{(}$such that the utility function is weakly concave is denoted by $U_{2}^{(} \cdot{ }^{2}$ This latter set of decisionmakers will be the main focus of our attention.

The types of problems that we intend to study might best be illustrated when there are just two assets. If $i^{\text {th }}$ asset location and scale parameters are given $r_{i}$ and $\mathrm{s}_{i}$, respectively, then the maximization problem may be written as

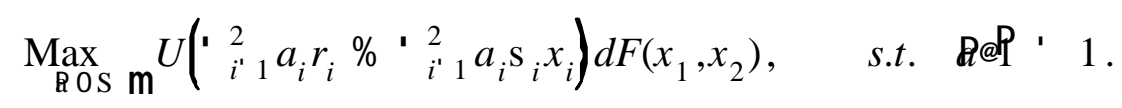

For this problem, we will pose three readily confirmed conjectures. If $r_{1}{ }^{\prime} r_{2}, \mathrm{~s}_{1}{ }^{\prime} \mathrm{s}_{2}$, and $F\left(x_{1}, x_{2}\right)$ is symmetric in the sense that $F\left(x_{1}, x_{2}\right) / F\left(x_{2}, x_{1}\right)$ then one might expect that $a_{1}^{(}{ }^{\prime} a_{2}^{(}$ú $U\left(@ O U_{2}^{(}\right.$. If $r_{1}>r_{2}, \mathrm{~s}_{1}{ }^{\prime} \mathrm{s}_{2}$, and $F\left(x_{1}, x_{2}\right) / F\left(x_{2}, x_{1}\right)$ then one might expect that $a_{1}^{(} \$ a_{2}^{(}$ú $U\left(@ O U_{1}^{(}\right.$. Finally, if $r_{1}{ }^{\prime} r_{2}, \mathrm{~s}_{1}>\mathrm{s}_{2}$, and $F\left(x_{1}, x_{2}\right) / F\left(x_{2}, x_{1}\right)$ then one might expect that $a_{1}^{(} \#$ $a_{2}^{(}$ú $U\left(@ U_{2}^{(}\right.$.

However, problems such as these become considerably more involved when $n>2$. To start with,

\footnotetext{
${ }^{1}$ Throughout, we will write vectors horizontally in order to conserve on space and notation.

${ }^{2}$ Emphasizing the algebraic nature of our approach, our analysis will have no need for the usual smoothness assumptions on $U(\mathrm{p})$.
} 
there are $n$ ! ways of interchanging $n$ arguments and the sorts of symmetries that $F\left(x_{1}, x_{2}\right)$ might possess also increases in a near exponential manner with the value of $n$. More importantly, unlike the symmetry assumption $F\left(x_{1}, x_{2}\right) / F\left(x_{2}, x_{1}\right)$, the sorts of symmetries that may arise when $n>2$ need not be reflections across a bisector. This is problematic because an analysis based on comparisons before and after reflections is often the most convenient line of approach. Fortunately, there exists a large body of mathematical tools that are quite well-suited for posing and systematically analyzing generalized versions of allocation order conjectures such as the three provided above. The goal of this paper is to bring these tools to an analysis of allocative order in the portfolio allocation problem.

\section{Methodological preliminaries}

Our interest in this paper is in the implications of symmetry for portfolio choice. One of the most general mathematical frameworks for characterizing symmetries is group theory. A related framework, one which places partial order on asymmetries between vectors, is generalized majorization theory. We will employ both, and this section describes the principal tools that will be applied.

\subsection{Groups}

A group is a set of operations that satisfies four convenient properties.

Definition 3.1. A group, $\tilde{G}$, is a set $G$ together with an operation * on $G$ such that each of the following axioms is satisfied

I) closure; $G$ is closed with respect to *,

II) associativity; $a\left(\left(b(c)^{\prime}(a(b)\right.\right.$ ( $c$ ú $a, b, c 0 G$, where the operations in parentheses occur first, III) existence of identity element; there is an $e 0 G$ such that $a\left(e^{\prime} e\right.$ ( $a^{\prime} a$ ú $a 0 G$, IV) existence of inverse elements; for each $g 0 G$ there exists a unique element, labeled $g^{\& 1} 0 G$, such that $g\left(g^{\& 1} g^{\& 1}\left(g^{\prime} e\right.\right.$.

The size of a group, the order, is given by the cardinality of set $G$. The sub-structure of a group is 
typically important when seeking to understand the group's implications.

Definition 3.2. A subgroup $\tilde{H}$ of group $\tilde{G}$ is a subset, $H$, of set $G$ that generates a group under the same operation *.

Notice that a group is a set of operations, and should not be confused with the set of objects on which it might act. In this paper, groups will act on a set of random variables by permuting the positions of these random variables in a multivariate distribution function. Because it is, perhaps, easiest to illustrate the concept of a group with reference to how it permutes objects, and because this is how we intend to apply groups, we will present the idea of a permutation group before providing some illustrations.

Definition 3.3. (Dixon and Mortimer, p. 5) Let $\mathrm{O}_{n}$ be a finite non-empty set of objects of cardinality $n$. A bijection of $\mathrm{O}_{n}$ onto itself is called a permutation of $\mathrm{O}_{n}$. The set of all such permutations forms a group under composition of mappings. This is called the symmetric group of $\mathrm{O}_{n}$, and is denoted by $\operatorname{Sym}\left(\mathrm{O}_{n}\right)$. Any subgroup of a symmetric group is called a permutation group. ${ }^{3}$

Thus, a permutation group is a formalization of the notion of a group acting on a set; in this case the set of objects represented by $\mathrm{O}_{n}$. From rudimentary combinatorics we know that the order of $\operatorname{Sym}\left(\mathrm{O}_{n}\right)$, denoted by $* \operatorname{Sym}\left(\mathrm{O}_{n}\right) *$, is $n !$.

Example 3.1. Pick an arbitrary distribution function representing three random variables, $F(\mathbf{P})$ $F\left(x_{1}, x_{2}, x_{3}\right)$. The symmetries, or invariances, of this function may form a group with order between 1

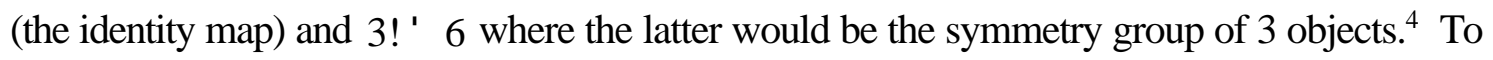
characterize a distribution with that order of symmetry, start with any primitive distribution

\footnotetext{
${ }^{3}$ It is for no other reason than presentation that the tilde is omitted when referencing the symmetric group, $\operatorname{Sym}\left(\mathrm{O}_{n}\right)$.

${ }^{4}$ In fact, by the Lagrange theorem, the order of any subgroup must divide the order of the group. And so a subgroup of the symmetries of $F\left(x_{1}, x_{2}, x_{3}\right)$ cannot be of an order other than 1,2, 3, or 6 .
} 
$\hat{F}(\mathbf{P})^{\prime} \hat{F}\left(x_{1}, x_{2}, x_{3}\right)$. Then define ${ }^{5}$

$$
\begin{aligned}
F(\boldsymbol{P}) ' & \frac{1}{6} \underset{g 0 \operatorname{Sym}\left(\mathrm{O}_{3}\right)}{\mathrm{j}} \hat{F}\left(x_{g(1)}, x_{g(2)}, x_{g(3)}\right) ' \frac{1}{6} \hat{F}\left(x_{1}, x_{2}, x_{3}\right) \% \frac{1}{6} \hat{F}\left(x_{2}, x_{1}, x_{3}\right) \\
& \% \frac{1}{6} \hat{F}\left(x_{3}, x_{2}, x_{1}\right) \% \frac{1}{6} \hat{F}\left(x_{1}, x_{3}, x_{2}\right) \% \frac{1}{6} \hat{F}\left(x_{2}, x_{3}, x_{1}\right) \% \frac{1}{6} \hat{F}\left(x_{3}, x_{1}, x_{2}\right)
\end{aligned}
$$

Representing the elements of the group in cycle notation, we have $e$ as the identity element,

$$
g_{1}{ }^{\prime}(1,2), g_{2}{ }^{\prime}(1,3), g_{3}{ }^{\prime}(2,3), g_{4}{ }^{\prime}(1,2,3), g_{5}{ }^{\prime}(1,3,2) \text {. }
$$

The group may be written in the form of a Cayley table as follows ${ }^{6}$

${ }^{5}$ Here, $(1,2)$ means that the first argument maps to the second and the second back to the first. Argument 3 is omitted because it is the convention in cycle notation not to list arguments that are fixed under the group element. Similarly, $g_{5}{ }^{\prime}(1,3,2)$ is to be read as the statement that the first argument maps to the third, the third to the second, and the second back to the first. Generally, $g(i)$ is the map of the $i^{\text {th }}$ object in $\mathrm{O}_{3}$ under $g 0 \operatorname{Sym}\left(\mathrm{O}_{3}\right)$. For example, $g^{\prime}(1,2,3)$ implies $g(1)^{\prime} 2, g(2)^{\prime} 3$, and $g(3) ' 1$.

${ }^{6}$ When interpreting the table, read $g_{i}\left(g_{j}\right.$ as $g_{i}$ after $g_{j}$ whereby $g_{3}\left(g_{2}{ }^{\prime} g_{4}\right.$ and $g_{2}\left(g_{3}{ }^{\prime} g_{5}\right.$. 
Table 1: Cayley table of $\operatorname{Sym}\left(\mathrm{O}_{3}\right)$

\begin{tabular}{c|cccccc}
$*$ * after & $e$ & $g_{1}$ & $g_{2}$ & $g_{3}$ & $g_{4}$ & $g_{5}$ \\
\hline$e$ & $e$ & $g_{1}$ & $g_{2}$ & $g_{3}$ & $g_{4}$ & $g_{5}$ \\
$g_{1}$ & $g_{1}$ & $e$ & $g_{5}$ & $g_{4}$ & $g_{3}$ & $g_{2}$ \\
$g_{2}$ & $g_{2}$ & $g_{4}$ & $e$ & $g_{5}$ & $g_{1}$ & $g_{3}$ \\
$g_{3}$ & $g_{3}$ & $g_{5}$ & $g_{4}$ & $e$ & $g_{2}$ & $g_{1}$ \\
$g_{4}$ & $g_{4}$ & $g_{2}$ & $g_{3}$ & $g_{1}$ & $g_{5}$ & $e$ \\
$g_{5}$ & $g_{5}$ & $g_{3}$ & $g_{1}$ & $g_{2}$ & $e$ & $g_{4}$
\end{tabular}

By construction, distribution $F(\mathbf{P})$ as given in (3.1) above is symmetric in any one of the group operations. Now let us take the cyclic subgroup of $\operatorname{Sym}\left(\mathrm{O}_{3}\right)$, so called because it cycles the three objects. Labeled $\tilde{C}_{3}$, it is comprised of $e, g_{4}{ }^{\prime}(1,2,3)$, and $g_{5}{ }^{\prime}(1,3,2)$. We may construct a distribution function that is symmetric, or invariant, under its elements as

$$
F(\ngtr) ' \frac{1}{3} \mathrm{j}_{g 0 \mathrm{C}_{3}} \hat{F}\left(x_{g(1)}, x_{g(2)}, x_{g(3)}\right) ' \frac{1}{3} \hat{F}\left(x_{1}, x_{2}, x_{3}\right) \% \frac{1}{3} \hat{F}\left(x_{2}, x_{3}, x_{1}\right) \% \frac{1}{3} \hat{F}\left(x_{3}, x_{1}, x_{2}\right)
$$

where $\hat{F}\left(x_{1}, x_{2}, x_{3}\right)$ is an arbitrary distribution. The Cayley table is

\begin{tabular}{|c|c|c|c|}
\hline$*=$ after & $e$ & $g_{4}$ & $g_{5}$ \\
\hline$e$ & $e$ & $g_{4}$ & $g_{5}$ \\
\hline$g_{4}$ & $g_{4}$ & $g_{5}$ & $e$ \\
\hline$g_{5}$ & $g_{5}$ & $e$ & $g_{4}$ \\
\hline
\end{tabular}

A function that is symmetric under the elements of group $\tilde{G}$ is said to be $\tilde{G}$-invariant so that function $F(\mathbb{P})$ in (3.2) is $\tilde{C}_{3}$-invariant. Clearly, $\tilde{C}_{3}$ is a subgroup of $\operatorname{Sym}\left(\mathrm{O}_{3}\right)$ because $F(\mathbb{P})$, as given in (3.1), is invariant under $\tilde{C}_{3}$ while $\tilde{C}_{3}$ is of lower order than $\operatorname{Sym}\left(\mathrm{O}_{3}\right)$. This particular subgroup 
retains a property of the symmetric group that will be of some importance in the present work. In both cases, the objects of the set orbit through the whole set, i.e., for any of the $x_{i}$ there exists an element of the group that will map the $x_{i}$ into any other $x_{j}$. That is, both groups are transitive. ${ }^{7}$

Definition 3.4. (Dixon and Mortimer, p. 8) A permutation subgroup of $\operatorname{Sym}\left(\mathrm{O}_{n}\right)$ is said to be transitive if the elements of $\mathrm{O}_{n}$ have only one orbit. A permutation subgroup that is not transitive is said to be intransitive.

Example 3.2. Excluding the group itself, there are four transitive permutation subgroups of $\operatorname{Sym}\left(\mathrm{O}_{4}\right)$. These include the cyclic group of order four, $\tilde{C}_{4}$. Indeed, any cyclic group is transitive as a consideration of $\tilde{C}_{4}$ will illustrate. In it, the four elements are $e, g_{1}{ }^{\prime}(1,2,3,4), g_{2}{ }^{\prime}(1,3)(2,4)$, and $g_{3}{ }^{\prime}(1,4,3,2) .^{8}$ To confirm transitivity, consider the second argument in a function. The group maps $e(2)^{\prime} 2, g_{1}(2)^{\prime} 3, g_{2}(2)^{\prime} 4$, and $g_{3}(2)^{\prime} 1$.

The group with elements $e, g_{1}{ }^{\prime}(1,2)(3,4), g_{2}{ }^{\prime}(1,3)(2,4)$, and $g_{3}{ }^{\prime}(1,4)(2,3)$ such that the Cayley table is

Table 3: Cayley table of a transitive subgroup of $\operatorname{Sym}\left(\mathrm{O}_{4}\right)$

\begin{tabular}{c|cccc}
$*=$ after & $e$ & $g_{1}$ & $g_{2}$ & $g_{3}$ \\
\hline$e$ & $e$ & $g_{1}$ & $g_{2}$ & $g_{3}$ \\
$g_{1}$ & $g_{1}$ & $e$ & $g_{3}$ & $g_{2}$ \\
$g_{2}$ & $g_{2}$ & $g_{3}$ & $e$ & $g_{1}$ \\
$g_{3}$ & $g_{3}$ & $g_{2}$ & $g_{1}$ & $e$
\end{tabular}

is also transitive. It is immediate from inspection of the group permutations that there exists a group

\footnotetext{
${ }^{7}$ Throughout this article, we rely heavily on Dixon and Mortimer (1996) for characterizations of transitive groups.

${ }^{8}$ In cycle notation, $g_{2}{ }^{\prime}(1,3)(2,4)$ is to be read as the pair of disjoint cycles $1: 3$ and $2: 4$.
} 
element that carries any object onto the position of any other object. For example, 262 under $e$, 261 under $g_{1}, 264$ under $g_{2}$, and 263 under $g_{3}$.

The group of order 8 with elements $e, g_{1}{ }^{\prime}(1,2,3,4), g_{2}{ }^{\prime}(1,3)(2,4), g_{3}{ }^{\prime}(1,4,3,2)$, $g_{4}{ }^{\prime}(1,3), g_{5}{ }^{\prime}(2,4), g_{6}{ }^{\prime}(1,4)(2,3)$, and $g_{7}{ }^{\prime}(1,2)(3,4)$ is also transitive. Notice that, since $\tilde{C}_{4}$ is a subgroup, the group must be transitive. The only other transitive subgroup of $\operatorname{Sym}\left(\mathrm{O}_{4}\right)$ is what is called the alternating subgroup, $\tilde{A_{4}}$. It has twelve elements, and $\tilde{C}_{4}$ is not a subgroup of $\tilde{A_{4}}$.

Example 3.3. An example of an intransitive subgroup of $\operatorname{Sym}\left(\mathrm{O}_{3}\right)$ is given in Table 4:

Table 4: Cayley table of an intransitive subgroup of $\operatorname{Sym}\left(\mathrm{O}_{3}\right)$

\begin{tabular}{c|cc}
$*=$ after & $e$ & $g_{1}$ \\
\hline$e$ & $e$ & $g_{1}$ \\
$g_{1}$ & $g_{1}$ & $e$
\end{tabular}

Here the non-identity group element is $g_{1}{ }^{\prime}(1,2)$, as in Example 3.1. The intransitivity is due to the absence of subgroup elements for the maps $x_{1} 6 x_{3}, x_{2} 6 x_{3}, x_{3} 6 x_{1}$, or $x_{3} 6 x_{2}$. The orbits of $x_{1}$ and $x_{2}$ are common, but this orbit is disjoint from that of $x_{3}$.

\subsection{Majorization}

Our concern is with realizations of vectors $\mathbb{P O S} \mathrm{f}^{\cdot}{ }^{n}$ and $\mathrm{PO} \mathrm{P}^{n}$. Mudholkar (1966) introduced the concept of group majorization pre-orderings of vectors in ${ }^{\prime} n$.

Definition 3.5. (Marshall and Olkin, p. 422) Let $\tilde{G}$ be a group of linear transformations mapping ${ }^{\cdot} n$ to ${ }^{\cdot}{ }^{n}$. Then $\mathrm{P}^{\mathrm{a}}$ is group majorized by $\mathrm{P}^{\beta}$ with respect to group $\tilde{G}$, written as $\mathrm{P}^{\mathrm{a}}$ ò $_{\tilde{G}} \mathrm{P}^{\beta}$, if $\mathrm{P}^{\mathrm{a}}$ lies in the convex hull of the orbit of $\mathrm{P}^{\beta}$ under $\tilde{G}$.

To illustrate, consider $\tilde{G}^{\prime} \quad \tilde{C}_{3}$ with elements $e, g_{4}$, and $g_{5}$ as given in Table 2. For $\mathrm{P}^{\mathrm{a}}$ ' 
$(0.5,0.3,0.2)$, the convex hull comprises of convex combinations of the vector set $\left\{P_{e}^{\mathrm{a}}, \mathbf{P}_{g_{4}}^{\mathrm{a}}, \mathbf{P}_{g_{5}}^{\mathrm{a}}\right\}$ ' $\{(0.5,0.3,0.2),(0.3,0.2,0.5),(0.2,0.5,0.3)\}$ so that $(0.4,0.25,0.35)$ is in the convex hull while $(0.6,0.2,0.2)$ is outside the convex hull. In the particular case where $\tilde{G}^{\prime} \operatorname{Sym}\left(\mathrm{O}_{n}\right)$, the group majorization pre-ordering is equivalent to the majorization as it is generally understood.

Definition 3.6. (Marshall and Olkin, pp. 67-68) For vectors $\mathbb{P O} 0^{\cdot n}$ and $\mathbb{P O}{ }^{\cdot n}$, denote the respective $k^{\text {th }}$ largest components as $u_{[k]}$ and $v_{[k]}$. Write $\mathbb{R}$ Ò P if a) ' ${ }_{k^{\prime}{ }_{1}}^{i} u_{[k]} \#^{\prime}{ }_{k^{\prime} 1}^{i} v_{[k]}$ ú $i 0$ $\mathrm{O}_{n \& 1}$, and b) ${ }^{\prime}{ }_{k^{\prime}}^{n} u_{[k]}{ }^{\prime} \quad{ }_{k^{\prime}}{ }^{\prime} v_{[k]}$. Then vector $\mathbb{P}$ is said to majorize vector $\mathbb{P}$.

\section{Diversification}

Before we establish our first main result, a lemma will prove to be both useful and insightful. ${ }^{9}$

Lemma 4.1. Let $U(\cdot) 0 U_{2}^{(}$. If $F(\mathbb{P})$ is $\tilde{G}$-invariant, then $\mathbb{P}^{(} \mathrm{ò}_{\tilde{G}} \mathbb{P}$ ú $\mathbb{P} O S$.

The lemma asserts that the optimal allocation vector of a risk averter is in the convex hull of the orbit of any $\mathbb{R O S}$ under the group of symmetries of the distribution function. Choosing an arbitrary $\mathbb{R O S}$, we may eliminate any $\boldsymbol{B}_{\tilde{G}} \ddot{O} \mathbb{R}$ without further consideration as a candidate for optimality. Note that the larger the order of the group the more discriminating the pre-order tends to be. For example, with $n$ quite large suppose that the only symmetry on $F(\boldsymbol{P})$ is the transposition $x_{1}: x_{2}$. Then the lemma can be viewed as generating convex hulls on an ${ }^{\cdot 1}$ subset of the ${ }^{\cdot} n \& 1$ dimensional simplex $S$. Consequently, little can be related about asset allocations other than about $a_{1}^{(}$and $a_{2}^{(}$. When the order of the group increases towards $n$ ! then more structure can be placed on the rankings throughout ROS.

The lemma proves to be particularly insightful when the orbit of $\mathrm{O}_{n}$ under the group is connected in the sense of being transitive.

Proposition 4.1. Let $U(\cdot) 0 U_{2}^{(}$. If the $\tilde{G}$-invariant group for $F(\mathbf{P}):^{\cdot}{ }^{n} 6{ }^{\cdot}$ is transitive on $\mathrm{O}_{n}$, then $\mathbb{P}^{(} \cdot(1 / n) \mathrm{P}$.

\footnotetext{
${ }^{9}$ Proofs are provided in the appendix.
} 
Observe that the Proposition does not assert $\mathbb{P}^{(} \mathrm{O}(1 / n) \mathbb{P}$ for intransitive permutation groups. Under an arbitrary intransitive group, $\mathbb{Z}^{(}{ }^{\prime}(1 / n) \mathrm{P}$ remains a possibility. But the existence of two or more orbits provides degrees of freedom for asymmetries to exist in $\mathbb{P}^{(}$.

Example 4.1. With $F(\mathbf{P})^{\prime} F\left(x_{1}, x_{2}, x_{3}, x_{4}\right)$, if the group of invariance is given by any of the four $\operatorname{Sym}\left(\mathrm{O}_{4}\right)$ subgroups outlined in Example 3.2 then Proposition 4.1 applies for all $U(\cdot) 0 U_{2}^{(}$.

However, with $F(\mathcal{P})^{\prime} F\left(x_{1}, x_{2}, x_{3}\right)$ invariant under the $\operatorname{Sym}\left(\mathrm{O}_{3}\right)$ subgroup as given in Example 3.3, then one may not be sure that $\mathbb{P}^{(} '(1 / n) \mathbb{P}$ under all $U(\cdot) 0 U_{2}^{(}$. All that can be ascertained from symmetry is that $a_{1}^{(} a_{2}^{(} \cdot{ }^{10}$

\section{Separability in allocation}

The question that we address in this section is the nature of the stochastic environment such that we can be sure how an optimizing agent allocates funds among a subset of all available opportunities. To do this, we need a sort of symmetric connectedness within the subset and, again, transitivity will do. We also need a form of conditional independence between subsets. The point-wise stabilizer subgroup suffices in this regard.

Definition 5.1. (Dixon and Mortimer, p. 13) Let $\tilde{G}$ be a permutation group on $\mathrm{O}_{n}$, and consider ? $\mathrm{f} \mathrm{O}_{n}$. The point-wise stabilizer of ?, as a subgroup of group $\tilde{G}$, is denoted by $\tilde{G}_{(\text {? })}$, and is given by the subgroup of $\tilde{G}$ such that each element of ? is held fixed. ${ }^{11}$

Example 5.1. Direct product groups have immediately identifiable subgroups that are clearly pointwise stabilizers of sets in a partition.

Definition 5.2. (Hungerford, p. 59) Let $\tilde{G}^{i}, i 0\{1,2, \ldots, I\}$, be a set of permutation groups with respective orders $n_{i}$. Form the Cartesian product $\times_{i^{\prime} 1}^{I} \tilde{G}^{i}$ with order $?_{i^{\prime} 1_{1} n_{i}}^{I}$. Then $\tilde{G}^{\prime} \times_{i^{\prime} 1}^{I} \tilde{G}^{i}$

${ }^{10}$ The equality arises from a simple application of the group majorization implications for concave functions that underpins Proposition 4.1.

${ }^{11}$ It is readily demonstrated that $\tilde{G}_{(?)}$ is a subgroup of $\tilde{G}$. 
forms a group under component-wise composition. This is called the direct product group.

For distribution function $F(\mathbf{P})^{\prime} F\left(x_{1}, x_{2}, \ldots, x_{6}\right)$, suppose that the symmetries of the first four arguments are as given in Example 3.2, Table 3 and label this group $\tilde{G}$. Also, let the last two arguments permute and label this group $\tilde{H}$ with elements $e_{H}$ and $h_{1}$. The direct product group $\tilde{Q}^{\prime} \tilde{G} \times \tilde{H}$ has eight elements, these being $e_{Q}{ }^{\prime}\left(e, e_{H}\right), q_{1}{ }^{\prime}\left(g_{1}, e_{H}\right), q_{2}{ }^{\prime}\left(g_{2}, e_{H}\right), q_{3}{ }^{\prime}\left(g_{3}, e_{H}\right)$, $q_{4}{ }^{\prime}\left(e, h_{1}\right), q_{5}{ }^{\prime}\left(g_{1}, h_{1}\right), q_{6}{ }^{\prime}\left(g_{2}, h_{1}\right), q_{7}{ }^{\prime}\left(g_{3}, h_{1}\right)$. The subgroup $\tilde{G} \times e_{H}$ fixes $x_{5}$ and $x_{6}$. The fixing is point-wise in that $x_{5}$ and $x_{6}$ do not permute in the subgroup.

An application of Lemma 4.1, together with Proposition 4.1, provides a form of weak separability on a partition of $\mathrm{O}_{n}$.

Proposition 5.1. Let $U(\cdot) 0 U_{2}^{(}$. For the group of symmetries, $\tilde{G}$, on distribution function $F(\mathbf{P})$, if $\tilde{G}_{\left(\mathrm{O}\left(\mathrm{O}_{i}\right)\right.}$ is transitive with respect to set $\mathrm{O}_{i}$, then $a_{j}^{(}{ }^{\prime} a_{k}^{(}$ú $j 0 \mathrm{O}_{i}$, ú $k 0 \mathrm{O}_{i}$.

Direct product groups are possessed of many well-structured point-wise stabilizer subgroups. In particular, as in Example 5.1, form a subgroup of $\tilde{G}^{\prime} \times_{i^{\prime} 1}^{I} \tilde{G}^{i}$ by choosing the identity in each $\tilde{G}^{i}$ except for some $i^{\prime} j 0\{1,2, \ldots, I\}$. And so we have a collection of stabilizer subgroups of $\tilde{G}$, one for each $\tilde{G}^{i}, i 0\{1,2, \ldots, I\}$. These subgroups are isomorphic, i.e., identical in structure, to the respective $\tilde{G}^{i}$ itself. This observation admits an extension of the proposition to generate a form of strong separability on a partition of $\mathrm{O}_{n}$.

Corollary 5.1. Let $U(\cdot) O U_{2}^{(}$. Form the partition $\left\{\mathrm{O}_{i}\right\}_{i^{\prime} 1}^{I}$ of $\mathrm{O}$, where $\operatorname{Sym}\left(\mathrm{O}_{i}\right)$ is the symmetric group of the $i^{\text {th }}$ set in the partition. Let $\tilde{G}^{i}$ be a subgroup of $\operatorname{Sym}\left(\mathrm{O}_{i}\right)$. If the group of symmetries, $\tilde{G}$, on distribution function $F(\mathbb{P})$ is given by $\times_{i^{\prime} 1}^{I} \tilde{G}^{i}$, and if each $\tilde{G}^{i}$ is transitive with respect to set $\mathrm{O}_{i}$,

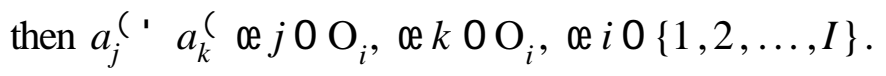

Example 5.2. For $\mathrm{O}_{5}$, let $\mathrm{O}_{a}{ }^{\prime}\{1,2\}$ and $\mathrm{O}_{b}{ }^{\prime}\{3,4,5\}$. If $\tilde{G}^{\prime} \operatorname{Sym}\left(\mathrm{O}_{a}\right) \times \tilde{C}_{3}$ where $\tilde{C}_{3}$ is the 
cyclic group on $\mathrm{O}_{b}$, then $a_{1}^{(}{ }^{\prime} a_{2}^{(}$and $a_{3}^{('} a_{4}^{(}{ }^{\prime} a_{5}^{(}$.

\section{Location shifts}

By contrast with the preceding sections, where asymmetries involved incompleteness in the symmetries of $F(\mathbb{P})$, this section and the section to follow will introduce and parameterize a second source of asymmetries between asset returns. In this section each of the random variables in $\boldsymbol{P}$ is held to have zero mean, but the true return is given by $\mathrm{P} \% \mathrm{P}, \mathrm{PO}{ }_{\%}^{n}$. Thus, location asymmetries are given by differences in the coordinate values of $P$. There remain also asymmetries that arise due to a small order, i.e., less than $n$ !, on the permutation group invariances of $F(\mathbf{P})$.

While attention in this section will be confined to $U(\cdot) 0 U_{1}^{(}$investors, and while we are now dealing with a new source of asymmetry, the notion of a convex hull remains central to our approach and to an understanding of our findings.

Proposition 6.1. Let $U(\cdot) 0 U_{1}^{(}$, and let $z_{i}{ }^{\prime} r_{i} \% x_{i}$ ú $i 0 \mathrm{O}_{i}$ where the $r_{i}$ are scalars and $\tilde{G}$ is the group of symmetries on $F(\mathbb{P})$. Then $\left(\mathbb{t}_{g}^{(} \& \mathbb{P}^{(}\right) \notin 0$ ú $g 0 G$.

Example 6.1. Suppose that distribution $F\left(x_{1}, x_{2}, x_{3}\right)$ is invariant under group $\operatorname{Sym}\left(\mathrm{O}_{3}\right)$, with $r_{1}$ $<r_{2}<r_{3}$. For the group operation $(1,2)$, i.e., transposition $x_{1}: x_{2}$, we have $\left(a_{1}^{(} \& a_{2}^{(}\right)\left(r_{1} \& r_{2}\right)$ $\$ 0$. For the group operation (2,3), i.e., $x_{2}: x_{3}$, we have $\left(a_{2}^{(} \& a_{3}^{(}\right)\left(r_{2} \& r_{3}\right) \$ 0$. Consequently, $r_{1}<r_{2}<r_{3}$ implies $a_{1}^{(} \# a_{2}^{(} \# a_{3}^{(}$. In general, if $r_{1}<r_{2}<\ldots<r_{n}$ and $F\left(x_{1}, \ldots, x_{n}\right)$ is invariant under $\operatorname{Sym}\left(\mathrm{O}_{n}\right)$, then $a_{1}^{(} \# a_{2}^{(} \# \ldots \# a_{n}^{(}$. The instance of Proposition 6.1 when $F\left(x_{1}, \ldots, x_{n}\right)$ is $\operatorname{Sym}\left(\mathrm{O}_{n}\right)$-invariant has been established by Lapan and Hennessy (2001).

Example 6.2. Consider the cyclic group $\tilde{C}_{3}$ on distribution $F\left(x_{1}, x_{2}, x_{3}\right)$ with $\mathrm{P}^{\prime}\left(r_{1}, r_{2}, r_{3}\right)^{\prime}$ $(1,2,3)$. Using the budget constraint, $a_{1}^{(} \% a_{2}^{(} \% a_{3}^{(} ' 1$, together with revealed preference condition $\left(\mathbb{R}_{g}^{(} \& \mathbb{P}^{(}\right) \notin \mathbb{Q}$ ú $g 0 G$, we have the portfolio allocation bounds 


$$
\mathrm{a} \$ a_{1}^{(}, \quad \mathrm{b} \$ a_{1}^{(} \% a_{2}^{(} \text {. }
$$

Example 6.3. A comparison of Examples 6.1 and 6.2 is instructive. Observe that ${ }^{*} C_{3}{ }^{* 1} 3<6{ }^{\prime}$ * $\operatorname{Sym}\left(\mathrm{O}_{3}\right)^{*}$. There is less symmetry to exploit in the cyclic group than in the corresponding symmetric group, and so the deductions concerning the optimal allocation vector should be no stronger.

Constraint set (6.1) does not allow us to assert that $a_{1}^{(} \# a_{2}^{(}$, and the partial nature of the ranking under $\tilde{C}_{3}$ would persist regardless of the values of $\left(r_{1}, r_{2}, r_{3}\right)$ so long as the $r_{i}$ are distinct. By contrast, group $\operatorname{Sym}\left(\mathrm{O}_{3}\right)$ generates a total ordering on the allocation vector. Notice too that, whatever $\tilde{G}$ in Proposition 6.1, the generated inequalities must bound the candidate allocation $\mathbb{A}^{(1}(1 / n) \mathbb{P}$. This is because the infinitely risk averse investor is in $U_{1}^{(}$.

The comparison between the examples is graphed in Figure 1. Inequality $a_{2}^{(} \# a_{3}^{(}$pertains under group $\operatorname{Sym}\left(\mathrm{O}_{3}\right)$, and this is least restrictive when $a_{2}^{(}{ }^{\prime} a_{3}^{(}$. This generates the bound $a_{1}^{(} \% 2 a_{2}^{(}{ }^{\prime} 1$. And we also have the bound $a_{1}^{(} \# a_{2}^{(}$. Together with the non-negativity constraints, these bounds define the inner hatched region. The larger hatched area is given when the distribution function is $\tilde{C}_{3}$ invariant. It can be seen that completing the symmetries shaves off two parts of the feasible set; namely below the diagonal but where $a_{1}^{(}<\mathrm{a}$, and above the diagonal but where $1 \& a_{2}^{(}<a_{1}^{(} \% a_{2}^{(}<\mathrm{b}$.

Example 6.4. For a 4-variate distribution function, suppose that the only symmetry is given by the reflection through the pair of hyperplanes $x_{1}{ }^{\prime} x_{3}$ and $x_{2}{ }^{\prime} x_{4}$ so that $F\left(x_{1}, x_{2}, x_{3}, x_{4}\right)^{\prime}$ $F\left(x_{3}, x_{4}, x_{1}, x_{2}\right)$. For $\left(r_{1}, r_{2}, r_{3}, r_{4}\right) '(1,3,4,5)$ then, together with the non-negativity constraints, the only non-trivial bound is $2 \% a_{3}^{(} \$ 5 a_{1}^{(} \% 4 a_{2}^{l}$.

Combining techniques employed in propositions 5.1 and 6.1, some work reveals the separability result

Proposition 6.2. Let $U(\cdot) 0 U_{2}^{(}$, and let $z_{i}{ }^{\prime} r_{i} \% x_{i}$ ú $i 0 \mathrm{O}_{i}$. For the group of symmetries, $\tilde{G}$, on 
$F(\mathbf{P})$, if $\tilde{G}_{\left(\mathrm{O}\left(\mathrm{O}_{i}\right)\right.}{ }^{\prime} \operatorname{Sym}\left(\mathrm{O}_{i}\right)$ then $\left(a_{j}^{(} \& a_{k}^{(}\right)\left(r_{j} \& r_{k}\right) \$ 0$ ú $j 0 \mathrm{O}_{i}$, ú $k 0 \mathrm{O}_{i}$.

Example 6.5. Consider group $\tilde{Q}$ as given in Example 5.1, and let $\mathrm{O}_{a}{ }^{\prime}\{5,6\}$. Then subgroup $\tilde{Q}_{\left(\mathrm{O}\left(\mathrm{O}_{a}\right)\right.}{ }^{\prime} e \times \tilde{H}$ is isomorphic to $\operatorname{Sym}\left(\mathrm{O}_{a}\right)$ so that the proposition yields $\left(a_{5}^{(} \& a_{6}^{(}\right)\left(r_{5} \& r_{6}\right) \$ 0$.

\section{Scale effects}

Whereas the focus of the last section was on the first moments of asset returns, we now seek a better understanding of how asymmetries in the second central moments of asset returns affect the allocation vector. To do so, we assume that mean returns are asset invariant, i.e., $r_{i}{ }^{\prime} \bar{r}$ ú $i 0 \mathrm{O}_{n}$, and we represent the magnitude of univariate risks faced by a vector of dispersion coefficients, $P$. Univariate returns are given by $z_{i}{ }^{\prime} \bar{r} a_{i} \% \mathrm{~s}_{i} a_{i} x_{i}, i 0 \mathrm{O}_{n}$. Defining $c_{i}{ }^{\prime} \mathrm{s}_{i} a_{i}$, we may write portfolio returns as

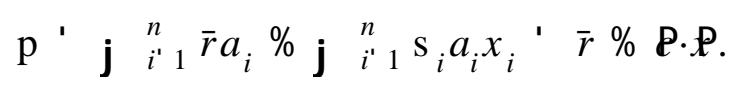

To exploit any group symmetries, $\tilde{G}$, on $F(\boldsymbol{P})$, we define the vector

$$
\mathbb{P}_{\mathrm{P}, g},\left(\frac{c_{g(1)}}{\mathrm{s}_{1}}, \frac{c_{g(2)}}{\mathrm{s}_{2}}, \ldots, \frac{c_{g(n)}}{\mathrm{s}_{n}}\right), \quad g 0 G
$$

The construct is of interest because the weightings $1 / \mathrm{s}_{i}$ normalize to generate an iso-risk contour for returns distributions that are invariant under $\tilde{G}$. To see this, let $\mathbb{P}^{\prime}\left(\mathrm{s}_{1} x_{1}, \mathrm{~s}_{2} x_{2}, \ldots, \mathrm{s}_{n} x_{n}\right)$ and write

$$
\begin{aligned}
& E\left[U\left(\bar{r} \% \mathbb{P}_{\mathrm{P}, g} \Phi\right)\right]^{\prime} \quad E\left[U\left(\bar{r} \% \%^{\prime}{ }_{i^{\prime} 1}^{n} \mathrm{~s}_{g(i)} a_{g(i)} x_{i}\right)\right] ' \quad E\left[U\left(\bar{r} \% \%^{\prime}{ }_{i^{\prime} 1}^{n} \mathrm{~s}_{i} a_{i} x_{g^{\& l}(i)}\right)\right]
\end{aligned}
$$

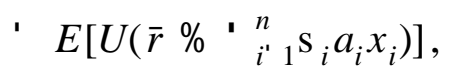

where the last inequality is due to the $\tilde{G}$ invariance of $F(\boldsymbol{P})$. But $\mathbb{P}_{\mathrm{P}, g}$, when viewed as an allocation vector, may not be feasible.

To develop an understanding of what $\mathbb{P}_{\mathbf{P}, g}$ are and are not feasible, and what choices reveal about 
feasibility, observe that, by construction, $\left[\mathbb{R}_{\mathbf{P}, g} /\left(\mathbb{R}_{\mathbf{P}, g} \cdot \mathbf{P}\right)\right] \cdot \mathbf{P} / 1$ is feasible. For future reference, define $\mathrm{d}_{g}{ }^{\prime} 1 /\left(\mathbb{P}_{\mathbf{P}, g} \cdot \mathbf{P}\right)$ with $\mathrm{d}_{g}^{(}{ }^{\prime} 1 /\left(\mathbb{P}_{\mathbf{P}, g}(\cdot \mathbf{P})\right.$. Returning to (7.2), and evaluating at the group identity,

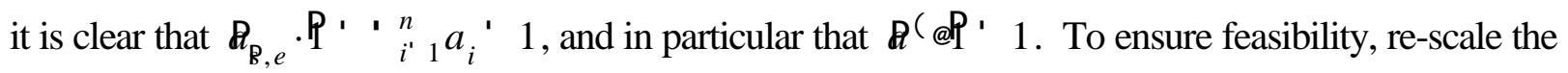
risk in (7.3) and evaluate at optimum choices to obtain

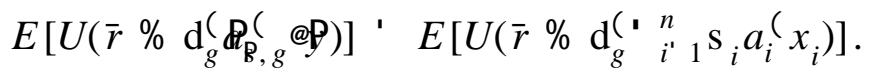

Because $U(\cdot) O U_{2}^{(}$and portfolio mean is invariant to the group operation, we must have $\mathrm{d}_{g}^{(} \$ \mathrm{~d}_{e}^{(}$ ú $g 0 G$, i.e., $\mathbb{P}_{\mathrm{P}, g}^{(} \cdot$ P \# 1 ú $g 0 G$. Relation $\mathrm{d}_{g}^{(} \$ \mathrm{~d}_{e}^{(}$asserts that no other allocation vector on the orbit of $\mathbb{P}_{\mathrm{P}, e}$ is as well diversified as $\mathbb{P}_{\mathrm{P}, e}^{(}$. Were this inequality not true, then the risk averse portfolio allocator would have optimized over available choices.

Proposition 7.1. Let $U(\cdot) 0 U_{2}^{(}$, let $z_{i}{ }^{\prime} \bar{r} a_{i} \% \mathrm{~s}_{i} a_{i} x_{i}$ ú $i 0 \mathrm{O}_{n}$, and define $\mathbb{R}_{\mathrm{P}, g}$ as in (7.2) above. For the group of symmetries, $\tilde{G}$, on $F(\mathbf{P})$, the optimal allocation vector must satisfy $\mathbb{P}_{\mathrm{P}, g}^{\mathrm{l}} \cdot \mathrm{P} \# 1$ ú $g 0 G$.

Example 7.1. Suppose that $\tilde{G}$ admits the simple transposition $x_{j}: x_{k}$. The proposition then asserts: if $\mathrm{s}_{j}>\mathrm{s}_{k}$, then $a_{k}^{(} \$ a_{j}^{(}\left(\mathrm{s}_{j} / \mathrm{s}_{k}\right) \$ a_{j}^{(}$. This particular case was demonstrated by Lapan and Hennessy (2001). For distribution $F\left(x_{1}, x_{2}, x_{3}\right)$, if $\tilde{G}^{\prime} \operatorname{Sym}\left(\mathrm{O}_{3}\right)$ and $\mathrm{P}^{\prime}\left(\mathrm{s}_{1}, \mathrm{~s}_{2}, \mathrm{~s}_{3}\right)^{\prime}(1,2,3)$ then application of the budget constraint delivers the constraint set $a_{1}^{(} \$ 2 a_{2}^{(}, 3 a_{1}^{(} \% 5 a_{2}^{(} \$ 3$, and $4 a_{1}^{(} \% 3 a_{2}^{(} \$ 3$ together with the non-negativity assumptions.

Example 7.2. Consider, as in Example 6.2, the cyclic group $\tilde{C}_{3}$ on distribution $F\left(x_{1}, x_{2}, x_{3}\right)$. This time however, let $\mathrm{P}^{\prime} \bar{r} \times \mathrm{P}$ and $\left(\mathrm{s}_{1}, \mathrm{~s}_{2}, \mathrm{~s}_{3}\right)^{\prime}(1,2,3)$. Employing budget constraint $a_{1}^{(} \% a_{2}^{(} \% a_{3}^{(')} 1$, as well as the two inequalities generated by the revealed preference deduction $\mathbb{P}_{\mathrm{P}, g}^{\mathrm{l}} \cdot \mathrm{P} \# 1$ ú $g 0 G$, we have the portfolio allocation bounds 


$$
12 \# 15 a_{1}^{(} \% 14 a_{2}^{(}, \quad 3 \# 7 a_{1}^{(} \& 3 a_{2}^{(} .
$$

Together with the assumption that $a_{3}^{(}>0$, these bounds are depicted in Figure 2. It is readily demonstrated that the bounds in Example 7.1 are tighter in that the admissible set for the optimal allocation vector is a subset of that identified in Figure 2. As in Example 6.3, this is because $\tilde{C}_{3}$ is a sub-group of $\operatorname{Sym}\left(\mathrm{O}_{3}\right)$.

It is also possible to establish a separability analog to the proposition.

Corollary 7.1. Let $U(\cdot) O U_{2}{ }^{(}$, let $z_{i}{ }^{\prime} \bar{r} a_{i} \%_{{ }_{i}} a_{i} x_{i}$ ú $i 0 \mathrm{O}_{n}$, and define $\mathbb{P}_{\mathrm{P}, g}$ as in (7.2) above. From the group of symmetries, $\tilde{G}$, on $F(\mathbb{P})$, establish the point-wise stabilizer of $\mathrm{O}\left(\mathrm{O}_{i}, \tilde{G}_{\left(\mathrm{O}\left(\mathrm{O}_{i}\right)\right.}\right.$. The optimal allocation vector must satisfy ' ${ }_{i 0 \mathrm{O}} a_{g}\left({ }_{g(i)} \mathrm{s}_{g(i)} / \mathrm{s}_{i} \#{ }^{\prime}{ }_{i 0 \mathrm{O}} a_{i}{ }_{i}\right.$ ú $g 0 G_{\left(\mathrm{O}\left(\mathrm{O}_{i}\right)\right.}$.

To ascertain the truth of this statement, observe that the order of the group tightens the bounds in Proposition 7.1. One is free, for the sake of convenience in analysis, to concentrate attention on subsets of the available investment opportunities. At, possibly, some loss in the strength of the bounds, the point-wise stabilizer subgroup can be used to draw attention to sectoral allocations.

Example 7.3. In Corollary 7.1, let there be five assets where the group invariances are as in Example 5.2. The $\tilde{C}_{3}$ subgroup is the point-wise stabilizer subgroup for $\mathrm{O}_{a}{ }^{\prime}\{1,2\}$. For this $\tilde{C}_{3}$ subgroup, the corollary asserts that $a_{4}^{(} \mathrm{s}_{4} \mathrm{~s}_{3}^{\& 1} \% a_{5}^{(} \mathrm{s}_{5} \mathrm{~s}_{4}^{\& 1} \% a_{3}^{(} \mathrm{s}_{3} \mathrm{~s}_{5}^{\& 1} \# a_{3}^{(} \% a_{4}^{(} \% a_{5}^{(}$and $a_{5}^{(} \mathrm{s}_{5} \mathrm{~s}_{3}^{\& 1} \% a_{3}^{(} \mathrm{s}_{3} \mathrm{~s}_{4}^{\& 1} \% a_{4}^{(} \mathrm{s}_{4} \mathrm{~s}_{5}^{\& 1} \# a_{3}^{(} \% a_{4}^{(} \% a_{5}^{(}$. For the $\operatorname{Sym}\left(\mathrm{O}_{a}\right)$ subgroup, the corollary's implication has already been established in Example 7.1.

\section{Reflection groups}

Let ${ }_{k} \mathrm{f} \mathrm{O}_{n}, k 0\{1,2\}$ where ${ }_{1-} ?_{2}{ }^{\prime} \mathrm{i}$ and where $*_{?}{ }_{1} *^{\prime} *_{?}{ }_{2} *^{\prime}$ ?. Among group operations, $g 0 G$, on $\mathrm{O}_{n}$ those such that $g ?_{1}{ }^{\prime} ?_{2}{ }^{\prime} g^{\& 1} ?_{1}$, and such that, without further loss of generality, the ordering of sets is preserved under the bijection, are particularly convenient for study. This is because 
reflections allow ready comparisons of evaluations through exploiting the separating hyperplane (i.e., a set of bisectors) along which the distribution function may be folded. In what is to follow, we will exploit such reflection subgroups for asset distributions that are differentiated by group symmetries on the distribution function of risk sources and also by location and scale vectors within the utility function. Assumption 8.1. For group $\tilde{G}$ on $\mathrm{O}_{n}$, let there exist a subgroup, $\tilde{H}$, of order 2 with $H^{\prime}(e, \hat{g})$.

Example 8.1. Group $\tilde{C}_{2 n \%}$ on $\mathrm{O}_{2 n \%}, n 0\{1,2, \ldots\}$ does not have any subgroups that satisfy Assumption 8.1 because no element is its own inverse. In fact, the group does not even have any subgroups. However, group $\tilde{C}_{2 n}$ on $\mathrm{O}_{2 n}$ does have a subgroup of order 2 . Writing ${ }_{1}{ }^{\prime}$ $\{n, n \& 1, \ldots, 1\}$ and $?_{2}{ }^{\prime}\{n \% 1, \ldots, 2 n\}$, there exists $\hat{g} 0 C_{n}$ such that $?_{1}: ?_{2}$. When $n^{\prime} 6$, the subgroup may be written in cycle notation as $\{e,(1,6)(2,5)(3,4)\}$, and $\hat{g}$ reflects through the set of bisectors $\left\{x_{1}{ }^{\prime} x_{6}, x_{2}{ }^{\prime} x_{5}, x_{3}{ }^{\prime} x_{4}\right\}$.

Example 8.2. In Example 3.2, a glance along the principal diagonal of Table 3 reveals three reflection subgroups of order 2 . Indeed, since $\hat{g}\left(\hat{g}^{\prime} e\right.$ in a reflection subgroup the presence of the identity (other than for $e\left(e^{\prime} e\right)$ on the principal diagonal is both necessary and sufficient for the existence of a reflection subgroup of order 2. Table 1 reveals three reflection subgroups for $\operatorname{Sym}\left(\mathrm{O}_{3}\right)$, while Table 2 shows that $\tilde{C}_{3}$ does not have any subgroups at all.

When there are reflection group symmetries on the distribution of randomness in returns, the consequences of both Proposition 6.1 and Proposition 7.1 may be strengthened. The approach is to apply these propositions, and then pair off each argument with its reflection.

Proposition 8.1. From the group of symmetries, $\tilde{G}$, on $F(\mathbb{P})$, let Assumption 8.1 pertain where the subgroup reflects some index subsets $?_{1}: ?_{2}$. If

a) $\quad U(\cdot) 0 U_{1}^{(}$and $z_{i}{ }^{\prime} r_{i} a_{i} \% a_{i} x_{i}$ ú $i 0 \mathrm{O}_{n}$, then õ $i 0 ?_{1}$ such that $\left(a_{\hat{g}(i)}^{(} \& a_{i}^{(}\right)\left(r_{\hat{g}(i)} \& r_{i}\right) \$ 0$. Furthermore, if $r_{\hat{g}(i)} \& r_{i}{ }^{\prime}$ a $<0$ ú $i 0 ?_{1}$ then ' ${ }_{i 0 ?} a_{i}{ }_{i} \$^{\prime}{ }_{i 0 ?{ }_{2}} a_{i}^{(}$. 
b) $U(\cdot) 0 U_{2}^{(}$and $z_{i}{ }^{\prime} \bar{r} a_{i} \%{ }_{i} a_{i} x_{i}$ ú $i 0 \mathrm{O}_{n}$, then õ $i 0$ ? ${ }_{1}$ such that $\left(\mathrm{s}_{\hat{g}(i)} a_{\hat{g}(i)}^{(} \& \mathrm{~s}_{i} a_{i}^{(}\right)\left(\mathrm{s}_{\hat{g}(i)} \& \mathrm{~s}_{i}\right) \# 0$. Furthermore, if $\mathrm{s}_{\hat{g}(i)} \& \mathrm{~s}_{i}{ }^{\prime} \beta<0$ ú $i 0 ?_{1}$ then ' ${ }_{i 0 ?} a_{i}{ }_{i} \#^{\prime}{ }_{i 0 ?}{ }_{2} a_{i}^{\prime}$.

The existence inferences assume greater consequences when the cardinality of $?_{1}$ (and so of $?_{2}$ ) is small. In particular, for $*$ ? ${ }_{1}{ }^{\prime} \quad 1$ then part b) implies: if $\mathrm{s}_{2}>\mathrm{s}_{1}$ then $a_{1}^{(} \$ a_{2}^{(}\left(\mathrm{s}_{2} / \mathrm{s}_{1}\right) \$ a_{2}^{(}$. Thus, Proposition 8.1 may be viewed as a generalization of the simple transposition that was studied in Example 7.1. Example 6.1 can also be seen to be an instance of the existence inference in part a) of the Proposition when $* ?{ }_{1}^{* 1} 1$. The portfolio sector results in parts a) and b) are due to the existence of a set of separating hyperplanes in parameter space. The set is $\left\{r_{\hat{g}(i)}{ }^{\prime} r_{i} \% \mathrm{a}: i 0 ?_{1}\right.$, a $\left.0{ }^{\circ}\right\}$ in part a) and $\left\{\mathrm{s}_{\hat{g}(i)}{ }^{\prime} \mathrm{s}_{i} \% \beta: i 0 ?_{1}, \beta 0^{\circ}\right\}$ in part b).

\section{Conclusion}

The intent of this paper has been to formalize, in a general manner, the modeling of asymmetries in the asset returns environment countenanced by an investor so as to better understand the ordinal and cardinal structure of the allocation vector. We readily acknowledge that our analysis is far from definitive, and we conclude with some conjectures concerning extensions.

Normal subgroups allow a factoring, or decomposition, of groups into groups of more manageable order. It is primarily for this reason that the subgroups of this form are central to many of the most important applications of group theory. They also possess strong relations with stabilizer groups. Perhaps portfolio separability results such as Proposition 5.1 and Corollary 5.1 could be extended if relevant subgroups were assumed to be normal?

A second conjecture pertains to an alternative approach to developing asymmetries in the returns distributions. Majorization with respect to a group provides a non-parametric treatment of asymmetries whereas the extension to include location and scale asymmetries in the marginals gives a parametric flavor to the latter part of our analysis. One might view the parameter vectors as an intuitive approach 
to constructing asymmetries. But there would appear to be no merit, other than the convenience of concreteness, to parameterize asymmetries. There exists a literature on using groups to "build" objects such as functions (Brown, 1989; Ronan, 1989). In the case of a bivariate distribution, Kijima and Ohnishi (1996) and Lapan and Hennessy (2001) have used the most elementary group, reflection through a line, to appended a functional asymmetry to a distribution function such that order could be induced on the optimal portfolio allocation. Perhaps, after some thought, this constructive approach may be extended to the $n$-variate context? The key may be to recognize the reflection operation as one that exploits the existence of a separating hyperplane, and to initiate a systematic approach to studying the consequences of separated convex sets. These sets might be in a parameter space, as in this paper, or where the elements are distribution functions. 


\section{References}

Atkinson, Anthony B.: On the measurement of inequality. Journal of Economic Theory 2, 244-263 (1970)

Brown, Kenneth S.: Buildings. Berlin: Springer-Verlag 1989

Brumelle, Shelby L.: When does diversification between two investments pay? Journal of Financial and Quantitative Analysis 9 (3, June), 473-483 (1974)

Chambers, Robert G., Quiggin, John: Uncertainty, production, choice, and agency. Cambridge UK: Cambridge University Press 1974

Dixon, John D., Mortimer, Brian: Permutation groups. Berlin: Springer 1996

Hadar, Josef, Russell, William R., Seo, Kae-Kun: Gains from diversification. Review of Economic Studies 44 (2, June), 363-368 (1977)

Hungerford, Thomas W.: Algebra. New York: Springer-Verlag 1974

Kijima, Masaaki, Ohnishi, Masamitsu: Portfolio selection problems via the bivariate characterization of stochastic dominance relations. Mathematical Finance 6 (3, July), 237-277 (1996)

Kijima, Masaaki: The generalized harmonic mean and a portfolio problem with dependent assets. Theory and Decision 43 (1, July), 71-87 (1997)

Landsberger, Michael, Meilijson, Isaac: Demand for risky financial assets: A portfolio analysis. Journal of Economic Theory 50 (1, February), 204-213 (1990)

Lapan, Harvey E., Hennessy, David A.: Symmetry and order in the portfolio allocation problem. Economic Theory, forthcoming (2001)

León, Ramón V., Proschan, Frank: An inequality for convex functions involving G-majorization. Journal of Mathematical Analysis and Applications 69 (2, June), 603-606 (1979)

Marshall, Albert W., Olkin, Ingram: Inequalities: Theory of majorization and its applications. San Diego: Academic Press 1979

McEntire, Paul L.: Portfolio theory for independent assets. Management Science 30 (8, August), 952-963 (1984) 
Mudholkar, Govind S.: The integral of an invariant unimodal function over an invariant convex set—An inequality and applications. Proceedings of the American Mathematical Society 17 (6, December), 1327-1333 (1966)

Ronan, Mark: Lectures on buildings. Boston: Academic Press 1989

Rothschild, Michael, Stiglitz, Joseph E.: Increasing risk I: A definition. Journal of Economic Theory 2 (September), 225-243 (1970)

Russell, Thomas, Farris, Frank: Integrability, Gorman systems and the Lie bracket structure of the real line. Journal of Mathematical Economics 29 (2, March), 183-209 (1998)

Saari, Donald G.: Mathematical structures of voting paradoxes: I. pairwise votes. Economic Theory 15 (1, January), 1-53 (2000a)

Saari, Donald G.: Mathematical structures of voting paradoxes: II. positional voting. Economic Theory 15 (1, January), 55-102 (2000b)

Salant, Stephen W., Shaffer, Greg: Unequal treatment of iddentical agents in Cournot equilibrium. American Economic Review 89 (3, June), 585-604 (1999)

Samuelson, Paul A.: General proof that diversification pays. Journal of Financial and Quantitative Analysis 2 (1, March), 1-13 (1967a)

Samuelson, Paul A.: Efficient portfolio selection for Pareto-Lévy investments. Journal of Financial and Quantitative Analysis 2 (2, June), 107-122 (1967b)

Sato, Ryuzo: Self-dual preferences. Econometrica 44 (5, September), 1017-1032 (1976)

Sharpe, William F.: Capital asset prices: A theory of market equilibrium under conditions of risk. Journal of Finance 19 (3, September), 425-442 (1965) 


\section{Appendix}

Proof of Lemma 4.1: The main thrust of this result is a special case of a finding due to León and Proschan (1979), which we demonstrate for the sake of completeness. Under $\tilde{G}$-invariance, the symmetry of the portfolio problem delivers

$$
E\left[U\left(\mathbb{P} \cdot \mathbb{P}_{g \& 1}\right)\right] ' \quad E\left[U\left(\mathbb{P}_{g} \cdot \mathbb{P}\right)\right] \quad \text { ú } g 0 G .
$$

Now consider a vector in the convex hull of the orbit of $\mathbb{R}$. With ${ }^{*} \tilde{G}^{* 1} m$, any point in the hull may be written as a convex combination of the $m$ points defined by $\mathbb{R}_{g_{i}}, g_{i} 0 G^{\prime} \quad\left\{g_{1}, g_{2}, \ldots, g_{m}\right\}$. Denumerate the group elements so that each has associated with it an element of $\{1,2, \ldots, m\}$. For weighting vector $\mathbf{P}^{\prime}\left(?_{1}, ?_{2}, \ldots, ?_{m}\right), ?_{i} 0[0,1],{ }^{\prime}{ }_{i^{\prime}}^{m} ?_{i}{ }^{\prime} 1$, define $\mathbb{B}^{\prime} \quad{ }^{\prime}{ }_{i^{\prime}}^{m} ?_{i} \mathbb{P}_{g_{i}}, g_{i} 0 G$. Now by Jensen's inequality, $U(\cdot) 0 U_{2}^{(}$, and the invariances under $\tilde{G}$ we have

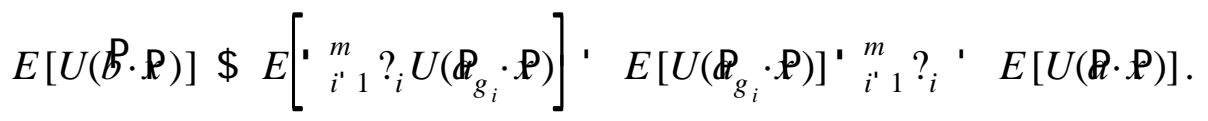

Therefore, $U(\cdot) O U_{2}^{(}$implies that $\boldsymbol{B}$ is weakly preferred over any $\mathbb{R O S}$. Next observe that $S \mathrm{f} \cdot{ }^{\cdot n}$ is convex. $S$ is closed under convex combinations and $\mathbb{P}^{\mathbb{P}}$ ò $_{\tilde{G}} \mathbb{P}$ ú $\mathbb{P O S}$.

Proof of Proposition 4.1: From Lemma 4.1, we know that the optimum is in the convex hull of the orbit under the group operations of all allocation vectors. Transitivity implies that there is just one orbit. Denote the convex hull of vector $\mathbb{R}$ under group $\tilde{G}$ by $C[\mathbb{R} ; \tilde{G}]$. Now $\left.\mathbb{B}_{\tilde{G}} \mathbb{R}\right]$ $C[\mathbb{B} ; \tilde{G}] f C[\mathbb{P} ; \tilde{G}]$ because $\mathbb{B}$ is inside the convex hull of $\mathbb{R}$ and $\mathbb{R}$ is outside the convex hull of $\mathbb{B}$. Finally, $C[(1 / n) \mathbb{P} ; \tilde{G}] f C[\mathbb{R} ; \tilde{G}]$ ú $\mathbb{P O S}$ because $C[(1 / n) \mathbb{P} ; \tilde{G}]$ is a singleton and no convex hull can be interior to it. Therefore, $\mathbb{P}^{(} \cdot(1 / n) \mathrm{P}$.

Proof of Proposition 5.1: Because $\tilde{G}_{\left(\mathrm{O}\left(\mathrm{O}_{i}\right)\right.}$ fixes all $i \mathrm{O} \mathrm{O}\left(\mathrm{O}_{i}\right.$ we may apply Lemma 4.1, but where 
the expectation in (A.1) is a conditional expectation over the random variables given by the index set $\mathrm{O}_{i}$ with all $x_{j}, j 0 \mathrm{O}\left(\mathrm{O}_{i}\right.$ fixed. The result then follows as in Proposition 4.1.

Proof of Proposition 6.1. Upon applying group elements,

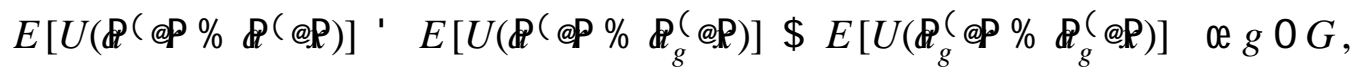

where the equality is due to group symmetries and the inequality is due to the fact that $\mathbb{P}^{(}$has been revealed to be weakly preferred over $\mathbb{P}_{g}^{(}$. But, due to group symmetries,

$$
E\left[U\left(\mathbb{P}_{g}^{(} \% \mathbb{P}_{g}^{(}\right)\right]^{\prime} \quad E\left[U\left(\mathbb{P}_{g}^{(} \% \mathbb{P}^{(} \Phi\right)\right] \quad \text { ú } g 0 G .
$$

Whence,

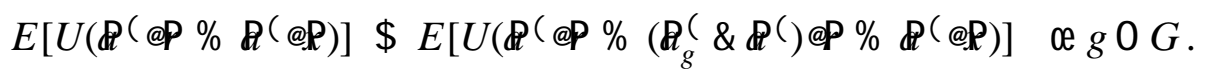

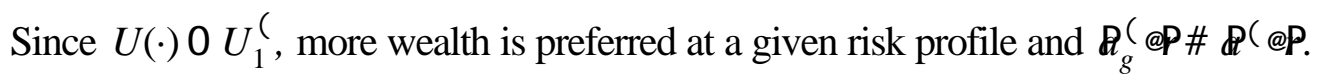

Proof of Proposition 6.2. As in Proposition 5.1, the transitivity of $\tilde{G}_{\left(\mathrm{O}\left(\mathrm{O}_{i}\right)\right.}{ }^{\prime} \operatorname{Sym}\left(\mathrm{O}_{i}\right)$ allows us to ignore $\mathrm{O}\left(\mathrm{O}_{i}\right.$. Now because $\tilde{G}_{\left(\mathrm{O}\left(\mathrm{O}_{i}\right)\right.}$ is the symmetric group on $\mathrm{O}_{i}$ it contains pair-wise transpositions. In particular, if we choose the group operation $x_{j}: x_{k}$ and we invoke the Proposition 6.1 finding that $\left(\mathbb{t}_{g}^{(} \& \mathbb{P}^{(}\right) \notin 0$, then $\left(a_{j}^{(} \& a_{k}^{(}\right)\left(r_{j} \& r_{k}\right) \$ 0$ follows.

Proof of Proposition 8.1.

Part a): Under the nonidentity element $\hat{g} 0 H$, using (A.5) above we will identify conditions under which $\mathbb{P}^{l} \pitchfork \mathbb{P}_{\hat{g}}^{l}$. This latter inequality may be expressed as 


$$
\mathrm{j} \quad{ }_{i 0 ?_{2}}\left(a_{\hat{g}(i)}^{(} \& a_{i}^{(}\right)\left(r_{\hat{g}(i)} \& r_{i}\right) \$ 0 .
$$

Therefore, some $i 0 ?_{1}$ must satisfy $\left(a_{\hat{g}(i)}^{(} \& a_{i}\left({ }^{\prime}\right)\left(r_{\hat{g}(i)} \& r_{i}\right) \$ 0\right.$. Next, if $r_{\hat{g}(i)} \& r_{i}{ }^{\prime}$ a $<0$ ú $i 0 ?{ }_{1}$ then (A.6) reduces to the assertion ' ${ }_{i 0 ?} a_{1} a_{i} \${ }^{\prime}{ }_{i 0 ?}{ }_{2} a_{i}$.

Part b): Applying Proposition 7.1 to the reflection subgroup in question, i.e., using the fact that ${ }_{1}$ and $?_{2}$ are separated to fold the reflection back on itself, we have ' ${ }_{i 00} a_{\hat{g}(i)}^{(} \mathrm{s}_{\hat{g}(i)} / \mathrm{s}_{i} \# 1$. Upon rearranging in the manner of (A.6) above, we have

$$
\mathrm{j} \quad{ }_{i 0 ?_{1}}\left(\mathrm{~s}_{\hat{g}(i)} a_{\hat{g}(i)}^{(} \& \mathrm{~s}_{i} a_{i}^{(}\right)\left(\mathrm{s}_{\hat{g}(i)} \& \mathrm{~s}_{i}\right) \# 0 .
$$

Therefore, some $i 0 ?_{1}$ must satisfy $\left(\mathrm{s}_{\hat{g}(i)} a_{\hat{g}(i)}^{(} \& \mathrm{~s}_{i} a_{i}^{(}\right)\left(\mathrm{s}_{\hat{g}(i)} \& \mathrm{~s}_{i}\right) \# 0$. Next, if $\mathrm{s}_{\hat{g}(i)} \& \mathrm{~s}_{i}{ }^{\prime} B<0$ ú $i 0 ?_{1}$ then (A.7) reduces to the assertion ' ${ }_{i 0} ?_{2} \mathrm{~s}_{i} a_{i}\left(\${ }^{\prime}{ }_{i 0 ?}{ }_{2} \mathrm{~s}_{i} a_{i}(\right.$. Finally, the positivity of the scale parameters together with a pairing off of reflected arguments and the assumption $\mathrm{s}_{\hat{g}(i)} \& \mathrm{~s}_{i}$ ' $\beta<0$ ú $i 0$ ? ${ }_{1}$ admit the weakening of ' ${ }_{i 0 ?_{2}} \mathrm{~s}_{i} a_{i}\left(\${ }^{\prime}{ }_{i 0 ?}{ }_{1} \mathrm{~s}_{i} a_{i}\right.$ to ' ${ }_{i 0 ?} a_{i}\left(\${ }^{\prime}{ }_{i 0 ?} a_{1}\right.$. 